\title{
Ethnologies
}

\section{Regards croisés : la collection du Musée de la civilisation}

\section{Andrée Gendreau}

Volume 24, numéro 2, 2002

\section{Musées}

Museums

URI : https://id.erudit.org/iderudit/006641ar

DOI : https://doi.org/10.7202/006641ar

Aller au sommaire du numéro

Éditeur(s)

Association Canadienne d'Ethnologie et de Folklore

ISSN

1481-5974 (imprimé)

1708-0401 (numérique)

Découvrir la revue

Citer cet article

Gendreau, A. (2002). Regards croisés : la collection du Musée de la civilisation. Ethnologies, 24(2), 107-124. https://doi.org/10.7202/006641ar

\section{Résumé de l'article}

Le visage de la collection du Musée de la civilisation a été dessiné par les collectionneurs, les gestionnaires et les conservateurs. Il nous fait part de regards croisés sur notre société. De regards qui varient selon les

collectionneurs, le lieu d'où ils observent, comprennent et analysent la société québécoise. La collection garde les traces des époques, de l'état d'avancement des connaissances, des objectifs poursuivis, et des moyens consentis pour la constituer. Elle est vivante, elle croît et se modifie avec l'histoire du Québec ; ce bref exposé racontera une partie de cet histoire. 


\title{
REgARDS CROISÉS : LA COLLECTION DU MUSÉE DE LA CIVILISATION
}

\author{
Andrée Gendreau \\ Directrice du Service des collections \\ Musée de la civilisation
}

Depuis les années 1980, les musées ont multiplié les études sur les représentations de l'Autre. Celles-ci s'inscrivent dans un courant d'analyse critique de l'anthropologie qui a remis en question les fondements même de cette discipline en l'associant aux politiques des nations impérialistes et aux attitudes méprisantes, voire arrogantes, des spécialistes. Les origines partagées, complexes et complices, des musées et de l'anthropologie, l'orientation publique prise par les musées au tournant des années 1950, au détriment de la recherche, privilégiée par les universités, mettaient les institutions muséales sous le feu des projecteurs. Ce qui fut appelé la «crise de la représentation» (Marcus et Fisher 1986: 8; Peressini 1999: 5) a également conduit à un questionnement sur l'autorité du locuteur. Qui a le droit de parler, comment et à quelles conditions? Bousculées par les critiques, souvent fondées, mais aussi très médiatisées et parfois tout simplement opportunistes, elles ont donné lieu à plusieurs réactions plus ou moins vives, variant de la pure peur panique à la rectitude politique, de la remise en question de nos pratiques de diffusion, en particulier des expositions, à celle du fonctionnement même de nos institutions. Ces dernières sont devenues des terrains expérimentaux pour une anthropologie plurielle (Peressini 1999: 7) tant au niveau du travail social sur les représentations, que sur la technique de représentation.

La grande majorité des études de représentation de l'Autre concerne les expositions, celles-ci constituant la première couche visible du travail de muséologie. Par l'analyse de la présentation et de la narration, on cherche à débusquer la perception des concepteurs, leur idéologie, leur vision du monde et de la place qu'ils détiennent dans l'univers culturel. 
Plusieurs raisons incitent à privilégier cet aspect de la muséologie pour les études de représentation. D'abord, les expositions s'adressent directement au public, elles poursuivent un double objectif : celui de vulgariser les connaissances et d'intéresser le public, de lui plaire, de le satisfaire. Or, pour éduquer et plaire, il faut non seulement être novateur, mais peut-être faut-il surtout élaborer un circuit expérimental articulé à une narration qui exprime un sens. Autrement dit, en plus de la clarté, de la justesse scientifique, il faut donner un sens à la vue, à la lecture, à l'émotion. Bien que cette strate visible du travail muséologique soit aujourd'hui la plus étudiée et la plus connue, elle est aussi la plus éphémère, en même temps qu'elle réclame et justifie les plus importants budgets, puisque c'est également elle qui fidélise le public et assure la défense des crédits. En fait, c'est celle qui s'inscrit le mieux dans une société médiatique, mondiale et de consommation. Il va donc de soi qu'alors que les expositions temporaires se multiplient, les expositions permanentes qui font appel aux collections des musées soient moins présentes. Il existe cependant, sous cette première strate, d'autres dimensions tout aussi importantes comme la conservation, la transmission, la recherche. Elles partagent même, avec l'éducation, l'un des fondements du musée comme institution publique.

La collection, quoique moins connue du public et moins visible que l'exposition, peut aussi servir d'objet d'étude des représentations. Sans doute moins directement explicite qu'une exposition, puisque celleci constitue un acte de communication directement destiné au public, une collection peut être tout aussi loquace sur les représentations du collectionneur et du musée, si l'on accepte de s'y attarder et de l'analyser.

À mon avis, on a accusé à tort la collection d'être le tombeau, voire le cimetière, de la culture vivante. Plutôt que de percevoir les objets de collection comme des reliques, il faut les considérer comme les éléments d'un corpus. Un corpus manipulable et capable de confirmer ou d'infirmer des hypothèses, un corpus qui nous éclaire sur les sujets de nos choix, selon notre cadre d'analyse.

Aujourd'hui je vous ferai part d'un visage particulier de la collection du Musée de la civilisation, celui qui a été dessiné par les collectionneurs, les gestionnaires et les conservateurs. Il nous fait part de regards croisés sur notre société. De regards qui se modifient selon le lieu d'où l'on observe, comprend et analyse la société, selon les objectifs poursuivis, selon l'état des connaissances et les moyens dont on dispose. Je ne 
toucherai pas à la collection autochtone, car il s'agit d'un cas particulier, d'un autre regard. Je me contenterai donc de raconter l'histoire d'une collection qui se voulait représentative du Canada, des Canadiens français, des Québécois (par cela il faut entendre Québécois francophones), puis qui se transforme de plus en plus pour ressembler aux citoyens actuels du Québec.

Pour faire d'une longue histoire une histoire courte, je me contenterai de relater les origines de la collection ethnographique nationale, en situant les principales acquisitions dans le temps. En second lieu, j'analyserai ces données par le prisme du point de vue porté sur l'histoire par les principaux acteurs. Je caractériserai ainsi la collection nationale par les types d'acquisition selon les objectifs de représentation. Que cherchait-on à illustrer par la collection? Quelles étaient les intentions explicites ou implicites derrière les acquisitions? En conséquence, que révèle la collection sur les idéologies qui l'ont colorée? Trois grandes tendances seront mises à jour : celle d'une vision romantique de la nation canadienne, suivie d'une approche nostalgique et nationaliste québécoise qui se mue, enfin, en un regard plus contemporain, nourri par les études historiques et sociologiques et par les grands débats de société sur la citoyenneté. Bien sûr, il s'agit d'un modèle ou d'une typologie qui, comme toute typologie, simplifie une réalité complexe. Les acteurs d'une collection nationale ne répondent pas tous aux mêmes critères en même temps. La vie, les occasions, les personnes et les conditions d'acquisition brouillent les images trop précises que présente le chercheur. Malgré tout, de grandes tendances se dégagent.

\section{Pour faire une histoire courte}

À sa création en 1920, le Musée de la province de Québec présente quatre grandes divisions : la peinture, la sculpture, les sciences naturelles et les « choses canadiennes " (Hamelin 1991: 28 ; Béland 1991) ${ }^{1}$. L'année 1927 signale le début officiel de la collection d'ethnographie du Gouvernement du Québec. Mais ce serait à la faveur de la Crise économique de 1929 que la collection de «choses canadiennes» démarre véritablement quoique, de toute évidence, celle-ci ne soulève pas d'enthousiasme. En effet, sous Pierre-Georges Roy, le premier directeur du Musée du Québec, la collection de «choses canadiennes ${ }^{2}$

1. Rappelons que l'évolution de l'appellation des Québécois de souche française passe de canadienne à canadienne-française pour aboutir à québécoise.

2. Jusqu'aux années 1940, le Musée du Québec désigne ainsi ses collections. 
croît maigrement et en marge des collections d'œuvres d'art. Au départ de son directeur, en 1941, le Musée compte à peine 200 pièces de cette collection embryonnaire. Pendant le mandat de Paul Rainville (19411952), la collection atteint presque 550 objets. Ce qui n'est guère mieux. C'est donc avec Gérard Morrisset (1953-1965) que le véritable envol aura lieu. Ce dernier doublera presque la collection, celle-ci atteignant environ 1000 pièces. Il s'agissait d'un rêve caressé depuis longtemps puisque déjà, en 1937, lors de l'Inventaire des oeuvres, il s'était donné comme mandat de réaliser le sauvetage de l'ensemble du patrimoine artistique, comprenant indistinctement l'art traditionnel, l'art populaire et l'artisanat. Les goûts personnels des responsables de ce projet ont cependant orienté relativement tôt la cueillette vers les arts décoratifs, excluant en grande partie les objets courants de la vie quotidienne. Aussi, lorsque Barbeau et Morrisset organisent les premières grandes expositions sur la culture matérielle, ils doivent emprunter plusieurs objets à des collections privées, dont la collection Coverdale. Bien que cette collection de "curiosités " n'ait pas encore trouvé son créneau, on doit à Gérard Morrisset d'avoir reconnu le patrimoine québécois comme digne d'intérêt et d'avoir participé à sa sauvegarde.

Cependant, c'est aux collectionneurs privés, majoritairement anglophones, que nous devons la véritable sauvegarde de notre patrimoine. Nous verrons qu'ils posent sur le patrimoine francophone un regard romantique et nationaliste canadien. La collection Coverdale est exemplaire à cet égard.

Parallèlement, l'industrialisation et la rapide urbanisation ont donné lieu à une prise de conscience des élites artistiques et intellectuelles francophones qui se retournent vers le passé mais pour y trouver "les débris des choses qui expriment l'âme canadienne-française » (Hamelin 1991: 29). Cette quête donnera lieu à un courant nostalgique et « ruraliste» qui marquera profondément la collection nationale. Voyons donc d'un peu plus près.

\section{William Coverdale, visionnaire au regard romantique}

Le cœur de la collection nationale aura été acquis en 1968, avec l'achat par le Gouvernement du Québec de la collection Coverdale, aussi appelée Tadoussac, à la compagnie Canada Steamship Lines. Rappelons que cette collection fut établie par la compagnie Canada 
Steamship Lines pour meubler et décorer l'hôtel Tadoussac et la maison Chauvin, voisine de l'établissement hôtelier. Selon Thérèse La Tour, conservatrice à l'Institut national de la civilisation, puis au Musée du Québec et enfin au Musée de la civilisation, l'acquisition de cette collection par le Gouvernement du Québec fut un «coup de maître » (La Tour : 1997). En effet, cette collection de près de 2500 objets est encore de nos jours le cœur et le fleuron de la collection nationale. Elle forme un ensemble représentatif des diverses fonctions aussi bien que des principaux caractères techniques, stylistiques et régionaux du mobilier traditionnel québécois. Environ 75 pièces ont été illustrées dans le célèbre ouvrage de Jean Palardy, Les meubles anciens du Canada français, sous la mention de Canada Steamship Lines. Cette collection contient en outre plusieurs autres ensembles d'objets tout aussi remarquables. Signalons au passage de la vaisselle d'étain, de la chaudronnerie, des faïences, des terres cuites et de la porcelaine, des luminaires, de la serrurerie, de la ferronnerie, des armes, entre autres.

Cette première grande acquisition sera rapidement suivie d'une seconde acquisition d'importance, celle de la Maison Chapais de SaintDenis de Kamouraska et de ses biens, un fonds d'objets et de documents fort éloquents sur l'univers d'une famille bourgeoise dont l'activité politique fut marquante pour les destinées du Québec. L'importance de ces acquisitions est telle qu'en 1969 on doit créer l'Institut national de la civilisation pour y veiller. Celui-ci est mandaté pour gérer la collection nationale et élaborer un projet de musée permettant sa mise en valeur. Par la suite, les acquisitions se poursuivront avec régularité, que ce soit par des ensembles ou par des objets individuels (voir le tableau des acquisitions). En 1983, après un débat houleux, le Musée du Québec, qui était responsable de la collection ethnographique depuis 1972, remet les 40000 pièces qui la composent au Musée de la civilisation. Aujourd'hui, la collection nationale comprend 225000 pièces, si l'on inclut le dépôt des prêtres du Séminaire de Québec.

Mais qui est donc ce William Hugh Coverdale ? Né en 1871 à Kingston, en Ontario, il s'éteint à New York en 1949. Il fut ingénieur, comme son père, qui était originaire d'une famille écossaise. Après avoir obtenu son baccalauréat en génie à Kingston, il poursuit ses études en Pennsylvanie où, après avoir travaillé une dizaine d'années pour une société ferroviaire, il adopte la citoyenneté américaine. En 1913, à New York, il s'associe à un Canadien, William Collpitts, pour fonder sa propre 


\section{Tableau 1}

Principales collections du Musée de la civilisation

\begin{tabular}{|c|c|c|}
\hline Collection & Nombre d'objets & Acquisition \\
\hline Collection Aimé-Desautels & $\begin{array}{l}605 \text { outils } \\
254 \text { poupées et accessoires }\end{array}$ & $\begin{array}{l}1996 \\
1992\end{array}$ \\
\hline $\begin{array}{l}\text { Collection des prêtres du } \\
\text { séminaire }\end{array}$ & $\begin{array}{l}\text { Dépôt d'environ } 80000 \text { objets : } \\
\text { beaux-arts, anthropologie, instruments } \\
\text { scientifiques, animaux naturalisés }\end{array}$ & 1995 \\
\hline Collection Henri-Dorion & 200 instruments de musique & 1994 \\
\hline Collection Pillon-Vallée & 1214 vêtements et accessoires & 1994 \\
\hline $\begin{array}{l}\text { Collection des Zouaves- } \\
\text { pontificaux-de-Québec }\end{array}$ & 550 objets & $1993-94$ \\
\hline Collection Claude-Davis & 2200 ornements de Noël & 1992 \\
\hline Collection Dominion-Corset & 125 sous-vêtements féminins & 1992 \\
\hline $\begin{array}{l}\text { Collection chinoise des } \\
\text { Jésuites }\end{array}$ & Plus de 2000 objets & 1990 \\
\hline Collection Rosario-Gauthier & 295 sculptures d'art populaire & 1988 \\
\hline $\begin{array}{l}\text { Fonds Jourdain-Fiset/Gabriel } \\
\text { Miller }\end{array}$ & 1145 meubles et accessoires & 1988 \\
\hline $\begin{array}{l}\text { Fonds Musée historique } \\
\text { canadien }\end{array}$ & $\begin{array}{l}341 \text { personnages et éléments de décor } \\
\text { du musée de cire }\end{array}$ & 1988 \\
\hline Collection Serge-Joyal & 3887 costumes et accessoires & 1987 \\
\hline $\begin{array}{l}\text { Collection du ministère de } \\
\text { l'Agriculture }\end{array}$ & $\begin{array}{l}\text { Plus de } 1584 \text { pièces : textiles, } \\
\text { céramiques, sculptures }\end{array}$ & $1983-2001$ \\
\hline Collection Lucie-Vary & $\begin{array}{l}600 \text { pièces, meubles, jouets, girouettes, } \\
\text { moules à sucre... }\end{array}$ & 1969 \\
\hline Collection Chapais-Barnard & $\begin{array}{l}\text { Univers d'une famille bourgeoise, } \\
1659 \text { pièces, meubles, photos... }\end{array}$ & 1968 \\
\hline Collection Coverdale & $\begin{array}{l}2500 \text { objets en ethnologie québécoise } \\
\text { et } 800 \text { objets d'origine autochtone }\end{array}$ & 1968 \\
\hline
\end{tabular}


société de génie civil. Il se marie l'année suivante, obtient un doctorat ès sciences cette même année et, huit ans plus tard, soit en 1922, il ajoute à son tableau d'honneur le grade de docteur en droit. Il présidera tout au cours de sa carrière la destinée de nombreuses compagnies, dont la Canada Steamship Lines de Montréal, pour qui il constituera la collection qui porte aujourd'hui son nom.

Cet Américain d'origine canadienne demeure probablement très attaché au pays de son enfance, puisqu'il y garde des liens, à la fois privés et publics. En effet, au-delà de l'homme d'affaires, président d'une compagnie canadienne de grande importance, il séjourne régulièrement avec sa famille dans sa résidence d'été ontarienne et il développe une affection particulière pour la région du Saguenay, où il se rend à plusieurs reprises pour ses affaires et dont il fera le sujet d'un livre publié en 1942, soit quelques années avant sa mort. Que nous dit cet ouvrage sur la vision qu'avait l'auteur du Canada et du Saguenay?

\section{Une vision romantique et politique}

The Kingdom of the Saguenay! A mysterious kingdom always kept apart from the rest of North America, an alluring region reached by the most mysterious stream of the the New World, a veritable fjord of immeasurable depth whirling out from between dark an gloomy cliffs rising sheer for thousands of feet from the sullen surface of the river! (Coverdale 1942: 7).

Voilà comment commence l'histoire du Saguenay. Après avoir raconté les petites et grandes histoires des autochtones, puis des pionniers Canadiens français y ayant séjourné, elle se termine par l'établissement de ce que l'auteur appelle un nouvel ordre, celui du Dominion du Canada et des États-Unis d'Amérique. Le chapitre qui en fait état s'intitule 1783 to 1942 Metamorphosis. On y lit que : "The new hotel (Hotel Tadoussac) is likewise a symbol that as the Indians slowly abandoned their old summer resort, Canadians and Americans redressed the balance » (19). Puis, un peu plus loin, l'auteur insiste sur l'établissement de ce nouvel ordre, opposant l'érection d'un hôtel de luxe au pourrissement de l'ancien poste de traite : " the new order at Tadoussac was symbolized by a grand hotel erected in 1864 near the decaying trading-post » (21). Enfin, il termine en vantant sa collection qui meuble les édifices érigés par la compagnie Canadian Steamship Lines : 


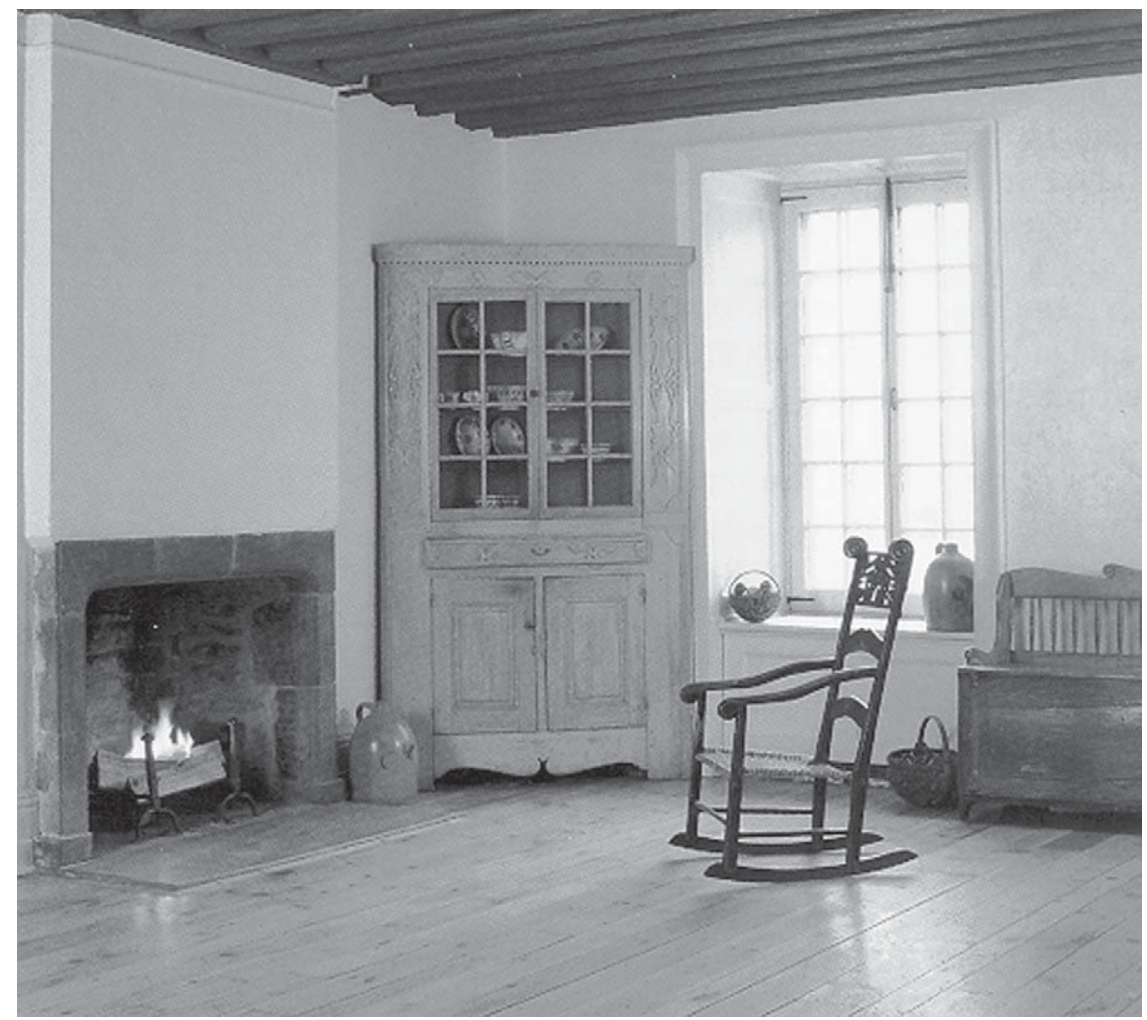

Figure 1. Collection Coverdale

Mobilier québécois

Banc-lit XIXe siècle, bois de pin autrefois peint.

Encoignure, Château-Richer, fin du XVIII e siècle, bois de pin autrefois peint.

For the «kingdom of the Saguenay » has truly become a veritable kingdom of delight. Shadowed by the rolling, forested Laurentian hills and facing the sweep of sparkling water-ways, this new, modern structure replaces the former hotel of the same name. It is furnished and decorated in «habitant " style and houses a priceless collection of early Canadian furniture and pictures (23).

Ces extraits expriment bien l'alliance que l'auteur établit entre les puissances canadiennes et américaines, le Dominion du Canada et les États-Unis, lesquels s'opposent à l'ancienne structure monarchique, ce « Royaume du Saguenay » si bien conservé à l'écart des grands courants nord-américains. 


\section{Etla collection?}

Normalement, si cette analyse était juste, la collection devrait faire état des dichotomies relevées dans le langage. Le fait-elle, et comment? Signalons tout d'abord que Coverdale a développé trois collections pour la compagnie Canadian Steamship Lines:

- une collection iconographique représentant le Canada. Celle-ci a été acquise par les archives nationales du Canada ;

- la collection ethnographique canadienne-française, comprenant environ 2500 pièces ;

- une collection ethnographique amérindienne et autochtone qui représente environ 50 nations avec 800 objets.

Or, non seulement les collections diffèrent-elles, mais les objectifs fondateurs aussi. Ainsi, alors que les objectifs de la collection iconographique appelée "Canadiana étaient explicitement pédagogiques et voulaient illustrer l'histoire du Dominion, ceux de la collection ethnographique étaient plus simplement pragmatiques : il s'agissait de meubler et de décorer l'hôtel Tadoussac avec bon goût en mettant en valeur un mobilier "rustique " très apprécié à l'époque par les américains et canadiens anglophones, tout en utilisant ce décor comme appât publicitaire : "We have perhaps well over one hundred items of this furniture, and our collection is constantly increasing as, in addition to its own use and beauty, we find it valuable for publicity purposes in that it creates great interest in all who see it " (Coverdale : 26 mars 1943).

Dans le but d'accroître les collections de la compagnie, Coverdale s'entoure donc de plusieurs intermédiaires, agents de la Canada Steamship Lines en poste au Canada et aux États-Unis, pour repérer des collectionneurs privés ou des musées possédant des artéfacts pouvant enrichir les collections en devenir. En ce qui concerne la collection de mobilier et d'objets domestiques, il avait aussi des acheteurs qui fréquentaient les encans ou faisaient de la réclame dans les journaux régionaux et locaux. De plus, il s'approvisionnait chez les antiquaires, principalement chez un certain Breiton, de Montréal. Ce dernier aurait été l'un des premiers, sinon le premier, antiquaire de mobilier canadienfrançais au Québec. D'origine russe, il a débuté sa carrière comme brocanteur. On disait alors «peddler». Il recherchait des meubles qui pouvaient répondre aux demandes du marché anglophone, principalement américain puisqu'il travaillait surtout pour un antiquaire 
de Boston. Le mobilier de style ou d'origine anglaise et américaine était donc retenu, alors que les meubles de pin, d'influence française, étaient systématiquement rejetés. Or, ceux-ci répondaient mieux aux besoins de Coverdale et au concept qu'il avait mis au point pour son hôtel de Tadoussac. Attentif au marché, Breiton n'a pas tardé à y voir son intérêt et quelques années plus tard il a ouvert boutique au centre-ville de Montréal (Sainte-Catherine et Université puis Peel). Une partie importante de la collection Coverdale serait attribuable à cet antiquaire clairvoyant. Jean Pallardy l'a d'ailleurs longuement interviewé dans le but d'établir la provenance régionale du mobilier de la collection.

\section{Un système de classification}

La correspondance du Président de la compagnie Canada Steamship Lines ou du conservateur de la collection ainsi que l'organisation matérielle des lieux de présentation des collections expriment bien les catégories, voire même les hiérarchies, établies entre les types de collections.

La correspondance : une double entrée

La correspondance laisse peu de doute quant à la hiérarchie des collections. Qu'elle soit signée par le Président ou par le Conservateur, la description des collections se présente toujours de la même façon: d'abord la collection de Canadiana, puis la collection canadiennefrançaise et « indienne ». Les descriptions des collections sont clairement divisées en deux paragraphes, le dernier associant toujours les collections canadienne-française et amérindienne. Même les termes utilisés illustrent cette hiérarchie :

«Canada Steamship Lines has, first of all, a collection of about 3,000 items of pictorial Canadiana, $(\ldots) »^{3}$ et il ajoute :

«In addition to this collection of Canadiana, we have much French Canadian furniture, fabrics, etc., and many relics of Indians and early French settlers at Tadoussac, which was established in the year 1600 » (Coverdale, correspondance, 26 mars 1943).

3. Je souligne. 


\section{Les lieux de présentation}

Les lieux de présentation sont aussi indicatifs du rapport aux collections. Ainsi, la collection iconographique est présentée au Manoir Richelieu, cet hôtel de luxe situé à Murray Bay, et qui était meublé de répliques de pièces de mobilier américain et anglais, alors que les deux autres collections sont logées à l'hôtel Tadoussac pour l'une et à la Maison-Chauvin (réplique du premier poste établi par les Français) pour l'autre.

Or, on a vu que l'hôtel Tadoussac, situé dans la sauvagerie et meublé en style "habitant ", illustre et témoigne de la supériorité du «nouvel ordre », imposé par les Canadiens et les Américains. Dans ce cadre, quelle est la position de la collection ethnographique canadiennefrançaise ? À mon avis, elle est ambiguë, n'ayant ni le statut éducatif de la collection iconographique, ni celui des reliques amérindiennes et françaises (French settlers at Tadoussac) qui servent à démontrer l'incontestable grandeur du nouvel ordre. Ambiguë parce qu'à la fois faisant partie de la représentation canadienne, mais témoignant en même temps d'un passé révolu, d'une période rustique, artisanale... habitante $»^{4}$ !

\section{Le style " habitant"}

Cette perception de la collection ethnographique comme témoignage de l'habitant du Québec est fort intéressante. On sait en effet que le mobilier et les objets de la collection Coverdale sont loin d'être des productions rustiques. Au moins la majorité des meubles de la collection ont été réalisés par d'habiles ébénistes artisans qui connaissaient les styles et les façons de faire.

Par ailleurs, une très grande partie des ustensiles de cuisine et de la ferronnerie provient d'Europe ou des États-Unis, quoique l'on suppose qu'ils aient été en usage au Québec. On croit cependant, mais sans pouvoir l'affirmer catégoriquement, que certaines pièces rares auraient été acquises directement en Europe ou aux États-Unis. Mais de toute façon, et de toute évidence, la collection Coverdale est plus représentative du grand mobilier québécois, du mobilier d'ébéniste, confectionné pour une classe relativement aisée. Le mobilier de style

4. On sait que le vocable « habitant » n'avait pas à l'époque le sens péjoratif qu'on lui prête aujourd'hui, mais il connotait tout de même un sens artisanal et rustique. 
« habitant» serait plutôt le résultat de la cueillette de la génération suivante, en particulier de ceux qui ont fait partie de ce que l'on appelle aujourd'hui l'école d'ethnologie de l'Université Laval.

\section{L'école d'ethnologie : une vision nostalgique et nationaliste}

Après la collection Coverdale, d'autres collections plus ou moins importantes ont suivi. Pour un moment gérée hors musée, la collection ethnographique s'est donc redéfinie autour d'un noyau dur constitué par une vision orientée vers le monde rural et les traditions folkloriques. Le programme d'ethnographie traditionnelle de l'Université Laval est

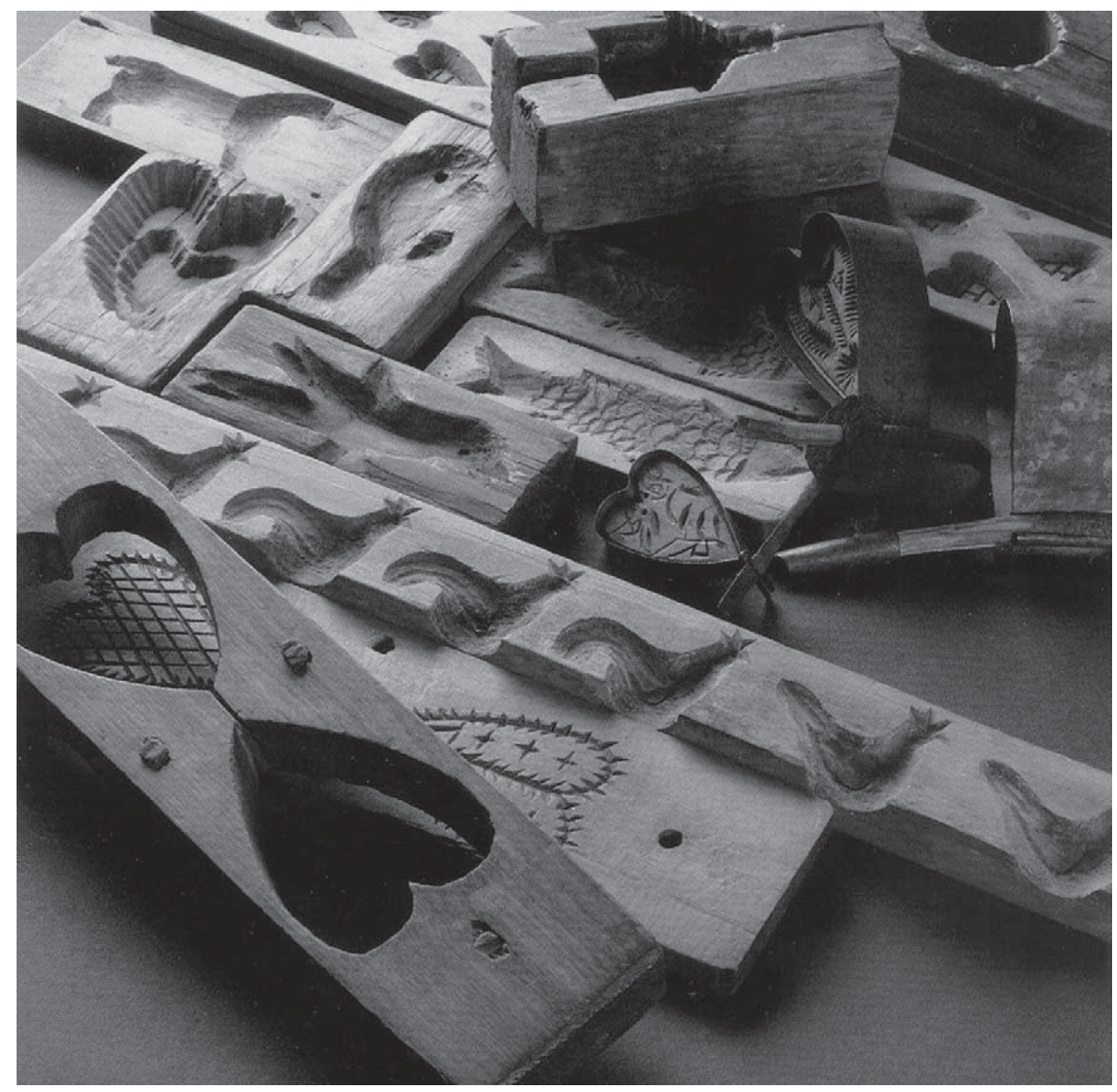

Figure 2. Moules à sucre.

Région de la Beauce et de Québec fin XIX ${ }^{\mathrm{e}}$ siècle.

On tire de ces moules à sucre en bois de pin, des oiseaux, des lapins, des castors, des poissons, des maisonnettes, des églises, mais surtout des coeurs de sucre décorés de motifs végétaux et astraux. 
alors très actif. On se préoccupe de culture matérielle et principalement de l'aspect comparatif des objets. Sous l'influence des ethnologues français André Leroi-Gourhan et Georges-Henri Rivière, on y développe des théories typologiques qui marqueront profondément les méthodes de collecte d'objets.

Bien que l'on s'éloigne d'une vision esthétique de la collection, on en conserve - sans doute inconsciemment - certains éléments, dont la pureté de l'objet et de son origine. On recherche surtout l'objet d'origine rurale, représentatif de la communauté francophone, l'objet particulier et unique si possible, mais surtout ancien, «le " plus ancien. On constitue des séries, chaque objet se distinguant par une modalité stylistique, le plus recherché étant le premier d'une série. Nous sommes encore au moment de la grande tentative de sauvetage du patrimoine rural et, comme cette période donne lieu à de nombreuses acquisitions, la collection nationale, toujours désignée sous le terme de «collection ethnographique », en conserve une empreinte large et profonde.

\section{Le Musée de la civilisation : une vision articulée à la réalité du Québec contemporain}

Le transfert de la collection ethnographique du Musée du Québec au Musée de la civilisation implique une nouvelle orientation. La collection nationale entre dans l'ère postmoderne. Tout en conservant l'intégrité et le sens des gestes des prédécesseurs, le Musée de la civilisation se donne comme mandat de moderniser la collection nationale. Comme pour les autres époques, elle doit rendre compte de son rapport avec la société actuelle et avec l'état d'avancement des connaissances dans le domaine de la muséologie. En d'autres termes, on doit poursuivre le développement de la collection à partir de ces considérations. Le Musée entend travailler sur le double front de la relecture de la collection ethnographique et de son développement dans une perspective plus contemporaine. À cette fin, il a défini ses priorités, ses principes et ses orientations de collectionnement. Ceuxci sont fondés sur quelques grands objectifs, dont l'obligation pour la collection de :

- constituer un témoin privilégié des cultures québécoise et francophone d'Amérique ;

- développer de nouvelles connaissances dans les domaines des cultures matérielle et sociale du Québec ;

- servir de support aux activités de recherche, d'éducation et de diffusion du Musée. 


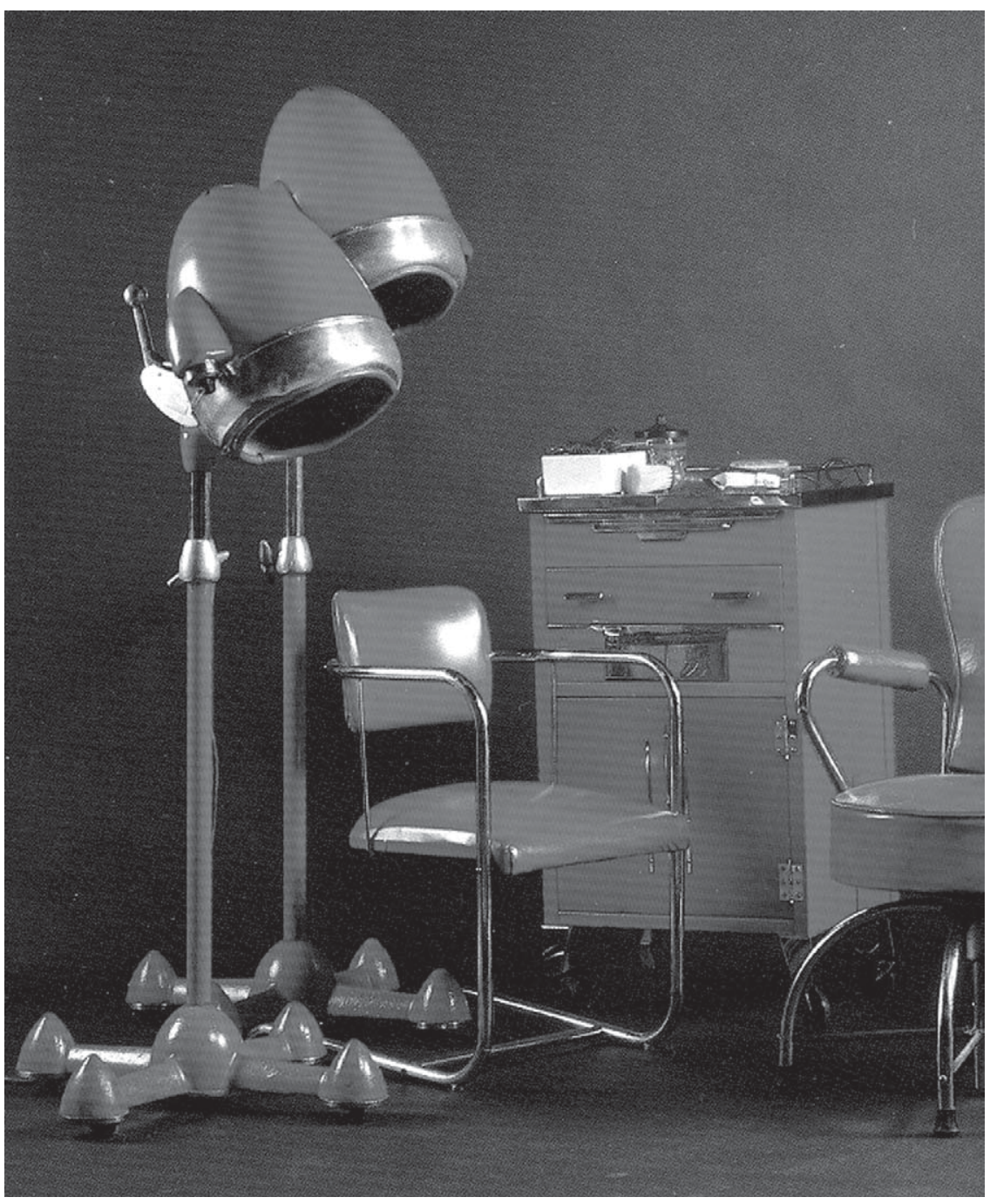

Figure 3. Salon de coiffure.

Provenance : Cap-de-la-Madeleine.

C'est durant les années 1930 que les coiffeuses des grandes villes, où elles exercent leur métier depuis une dizaine d'années, commencent à attirer les femmes des campagnes. 
Tableau 2

Les axes de développement de la collection

\begin{tabular}{|c|c|c|}
\hline $\begin{array}{l}\text { Axes de développement } \\
\text { absents ou presque dans } \\
\text { la collection }\end{array}$ & Nouveaux axes à développer & Remarques \\
\hline $\begin{array}{l}\text { 1. la représentation de la } \\
\text { diversité culturelle au } \\
\text { Québec }\end{array}$ & $\begin{array}{l}\text { - Peuples constitutifs } \\
\text { - Immigrants }\end{array}$ & $\begin{array}{l}\text { Il s'agit de mieux représenter les citoyens du Québec } \\
\text { en s'ouvrant aux peuples constitutifs de la nation : } \\
\text { Anglais, Écossais, Irlandais et à l'immigration récente. }\end{array}$ \\
\hline $\begin{array}{l}\text { 2. L'environnement et la } \\
\text { santé }\end{array}$ & $\begin{array}{l}\text { - Habitat (adaptation à l'hiver) } \\
\text { - Métiers de la santé, etc. }\end{array}$ & $\begin{array}{l}\text { Le développement du domaine de la santé doit se faire } \\
\text { dans le respect des mandats des autres institutions } \\
\text { spécialisées dans ce domaine. }\end{array}$ \\
\hline $\begin{array}{l}\text { 3. Les grandes figures ou } \\
\text { les destins particuliers }\end{array}$ & $\begin{array}{l}\text { Figures politiques, sociales, } \\
\text { littéraires, etc. }\end{array}$ & $\begin{array}{l}\text { Les objets significatifs des activités spécifiques ou } \\
\text { engagées des figures marquantes de notre histoire. }\end{array}$ \\
\hline $\begin{array}{l}\text { 4. Les loisirs, le sport et } \\
\text { les arts d'interprétation }\end{array}$ & $\begin{array}{l}\text { - Sport et loisir (chasse et } \\
\text { pêche, sports en vogue) } \\
\text { - Arts de la scène }\end{array}$ & $\begin{array}{l}\text { Notre collection est particulièrement pauvre à cet égard. } \\
\text { Le loisir faisant partie autant de la tradition que de la } \\
\text { vie contemporaine, il faudrait en tenir compte. }\end{array}$ \\
\hline $\begin{array}{l}\text { 5. La présence québé- } \\
\text { coise à l'étranger : les } \\
\text { aventuriers du coeur et } \\
\text { de l'esprit }\end{array}$ & $\begin{array}{l}\text { - Missionnaires } \\
\text { - Scientifiques } \\
\text { - Coopérants }\end{array}$ & $\begin{array}{l}\text { À cet égard, les objets recueillis dans le cadre des } \\
\text { missions sont privilégiés. Pensons à des collections } \\
\text { anthropologiques, à des objets utilisés par des } \\
\text { coopérants lors de leurs travaux, à des collections } \\
\text { missionnaires ou encore à des objets reçus comme } \\
\text { marque de reconnaissance. (Il faudrait éviter ce qui se } \\
\text { rapproche des "souvenirs de voyage"). }\end{array}$ \\
\hline Mobilier & $\begin{array}{l}\mathrm{XX}^{\mathrm{e}} \text { siècle, art nouveau et } \\
\text { mobilier courant de culture } \\
\text { populaire }\end{array}$ & Les autres époques sont à consolider. \\
\hline Vêtement & $\begin{array}{l}\text { Vie quotidienne, activités } \\
\text { sportives, associations, } \\
\text { professions, enfants, hommes }\end{array}$ & $\begin{array}{l}\text { Une politique et un programme de travail cibleront des } \\
\text { actions. }\end{array}$ \\
\hline Métiers et professions & $\begin{array}{l}\text { Métiers du } \mathrm{XX}^{\mathrm{e}} \text { siècle, métiers } \\
\text { importants non représentés : le } \\
\text { droit, le cinéma, la télé, le } \\
\text { journalisme, etc. }\end{array}$ & $\begin{array}{l}\text { La collection doit être actualisée. Il faut également tenir } \\
\text { compte des outils de transition. }\end{array}$ \\
\hline Objets de divertissement & Voir arts d'interprétation & \\
\hline Inuit & $\begin{array}{l}\text { Cibler des gravures ou des } \\
\text { sculptures qui font état de } \\
\text { l'évolution des tendances } \\
\text { contemporaines. Compléter les } \\
\text { parties manquantes par des } \\
\text { oeuvres acquises auprès des } \\
\text { missionnaires. }\end{array}$ & Voir la politique de développement de cette collection. \\
\hline Amérindiens & $\begin{array}{l}\text { Production actuelle chez les } \\
\text { Atikamekw et les Algonquins. } \\
\text { Poursuivre avec circons- } \\
\text { pection les grandes forces de } \\
\text { la collection Coverdale. }\end{array}$ & Voir la politique de développement de cette collection. \\
\hline
\end{tabular}


Afin de bien répondre au premier objectif, une démarche a été entreprise dès 1988 pour identifier et définir les lieux d'investissement du travail de collection et de conservation. Ce processus de rationalisation du processus de collectionnement se poursuit toujours. Plus récemment, nous avons publié un document faisant état des grandes orientations de la collection nationale. Parmi les grands axes de développement de la collection qui ont été retenus (voir tableau 2), on notera sans doute l'affirmation d'une volonté d'ouverture à l'Autre, en particulier au plan international et à celui des diversités culturelles au Québec. Nous en sommes actuellement à élaborer un plan stratégique de collectionnement. Par ces orientations et par les gestes qui en découlent nous souhaitons conserver l'intégrité et la signification de la collection que nous avons reçue en héritage d'une part et, d'autre part, mieux articuler la collection nationale aux réalités contemporaines du Québec.

\section{Conclusion}

On a vu que les objectifs et les visions des grands collectionneurs varient selon les époques et les réalités sociales et politiques. Dans ce sens, les collections et leurs archives forment un corpus original pour mieux comprendre les motivations qui ont guidé le choix des collectionneurs et leur perception de la société productrice des artéfacts ainsi collectionnés.

Si les collections nous renseignent sur les collectionneurs, elles nous informent également sur la société productrice et usagère des artéfacts. Ainsi, la collection ethnographique fait état de la présence de classes sociales différenciées au Canada français, de classes aisées et de classes plus pauvres. Elle nous rappelle également que l'ouverture aux produits extérieurs a existé depuis les tous débuts de la Nouvelle-France, les objets nous parvenant non seulement de France et d'Europe, mais d'Asie et des États-Unis. Elle nous fait également part du regard des intellectuels sur le monde rural, un regard nostalgique qui recherche la pureté des origines. Par ailleurs, on ne peut s'empêcher de noter que la collection est remarquablement centrée sur le Québec francophone. L'absence des communautés constitutives du Québec ne peut qu'être douloureuse et demander correction. Par ailleurs les aléas de l'histoire font que les anciennes cultures autochtones et inuites sont mieux documentées dans les collections européennes que dans les nôtres... Le hasard ne peut à lui seul combler les lacunes. Pour assurer une véritable représentativité à la collection nationale du Québec, il faut une volonté d'enrichissement 
de nos collections selon les besoins spécifiques de chacune des communautés et l'élaboration d'une planification rigoureuse des acquisitions. 


\section{Références}

Arpin, Roland, 1998, Le Musée de la civilisation. Une histoire d'amour, Collection Images de sociétés, Québec, Musée de la civilisation / Fides.

Béland, Mario, 1991, Musée du Québec. Les expositions des origines à 1990, Québec, Musée du Québec.

Cauchon, Sylvie et al., 1990, Objets de civilisation, Québec, Musée de la civilisation, Éditions Broquet Inc.

Coverdale, William Hugh, 1942, Tadoussac Then and Now. A History and Narrative of the Kingdom of the Saguenay, Canada, William Hugh Coverdale.

__, 26 mars 1943, "Correspondance », Dossier documentaire de la collection de la Canada Steamship Lines, Québec, Musée de la Civilisation.

Dubé, Richard, 1998, Trésors de société. Les collections du Musée de la civilisation, Québec, Collection Images de sociétés, Musée de la civilisation / Fides.

Gendreau, Andrée, 2002, La collection du Musée de la civilisation. Principes et Orientations, Québec Service des collections, Musée de la civilisation.

Hamelin, Jean, 1991, Le Musée du Québec. Histoire d'une institution, Québec, Musée du Québec.

La Tour, Thérèse, 1997, L'histoire des collections, document administratif, Québec, Service des collections, Musée de la civilisation.

Les axes de développement de la collection, 1989, document administratif, Québec, Musée de la civilisation, Service des collections.

Marcus George E. et Michael M. J. Fisher, 1986, Anthropology as Cultural Critique: An Experimental Moment in the Human Sciences, Chicago, University of Chicago Press.

Mission, concept et orientations - Musée de l'Amérique française, 1998, Québec, Musée de la civilisation.

Mission, concept et orientations - Musée de la civilisation, 1996, Québec, Musée de la civilisation, Muséotraces - Recherche et collections, Musée de la civilisation, Vol. 1 (4), octobre.

Peressini, Mauro, 1999, «Anthropologie et musées, introduction » dans Anthropologica vol. XLI (1), Société canadienne d'anthropologie : $3-12$. 